\title{
The Effect of Dual Trigger of Oocyte Maturation with Gonadotropin-Releasing Hormone Agonist and Low-Dose Human Chorionic Gonadotropin on Pregnancy Rate in Women at Risk of Ovarian Hyperstimulation Syndrome Marwa Salah Elsayed Abdel-Aal ${ }^{1 *}$, Yehia Abdel Salam Wafa ${ }^{1}$, Shokry Abdel Azeim El Shershaby ${ }^{2}$ \\ ${ }^{1}$ Obstetrics and Gynecology Departments, Faculty of Medicine \\ ${ }^{2}$ International Islamic Center for Population Studies and Research (Assisted Reproduction Unit) Al-Azhar University \\ *Corresponding author: Marwa Salah Elsayed Abdel-Aal, Registrar OB/GYN, MOH, Egypt, Phone: 020/01065117808; E-mail: marwasalah1612@gmail.com
}

\begin{abstract}
Background: the purpose of this study was to compare rates of ovarian hyperstimulation syndrome (OHSS) and the pregnancy outcome after using gonadotropin-releasing hormone agonists (GnRHa) alone and GnRHa in combination with low-dose human chorionic gonadotropin (hCG, dual trigger) for final oocyte maturation in women undergoing controlled ovarian hyperstimulation $(\mathrm{COH})$.

Patients and Methods: the study included 150 female patients with high risk of OHSS occurrence who were allocated by the Computer-based randomization method into two groups with 75 patients in each arm, Group I received GnRHa trigger and Group II received dual trigger (GnRHa + low-dose (1500 IU) hCG trigger).

Results: Our study showed that the incidence of OHSS was higher after dual trigger than GnRHa trigger but with no statistically significant difference ( $8.0 \mathrm{vs} 1.33 \%, \mathrm{p}>0.05)$. There were 6 cases of OHSS developed with dual trigger group (Group II) (3 were mild early OHSS, 2 were moderate early and one case was severe late OHSS). In contrast, there was only one case of severe late OHSS seen in Group I.

Conclusion: Dual trigger for final oocyte maturation using GnRHa and low-dose hCG is associated with increased the incidence of early OHSS compared to GnRH alone. However, dual trigger appears to be a safe approach with a satisfactory pregnancy outcome. Also, the dual trigger improves the oocyte maturation, the number of yield embryos and the quality of embryos.

Keywords: Dual trigger, gonadotropin-releasing hormone agonist, human chorionic gonadotropin, ovarian hyperstimulation syndrome, luteal phase support, oocyte maturation, quality of embryos.
\end{abstract}

\section{INTRODUCTION}

Ovarian hyperstimulation syndrome (OHSS) is an iatrogenic complication of supra-physiologic ovarian stimulation. The syndrome is almost exclusively associated with exogenous gonadotropin stimulation and is only rarely observed after clomiphene citrate treatment or spontaneous ovulation ${ }^{(1)}$.

OHSS characterized by cystic enlargement of the ovaries and acute fluid shift from the intravascular space to the third space caused by an increase in vascular permeability and neoangiogenesis. The clinical manifestations of OHSS reflect the extent of the shift of fluid into the third space and the resulting hemoconcentration due to intravascular volume depletion. Symptoms range from mild abdominal distention due to enlarged ovaries alone or with an accompanying fluid shift into the abdomen, to renal failure and death as a result of hemoconcentration and reduced perfusion of organs such as the kidneys, heart and brain (2).

The exact cause of OHSS has not been completely elucidated, but it seems likely that the release of vasoactive substances secreted by the ovaries under human Chorionic Gonadotrophin (hCG) stimulation may play a key role in triggering this syndrome ${ }^{(3)}$. Standard treatments for OHSS are generally conservative, and potentially lifethreatening complications of OHSS, which require costly long-term hospitalizations, render prophylactic measures a must ${ }^{(4)}$.

Many strategies have been tried to prevent OHSS, including cycle cancellation, coasting (5), intravenous albumin administration around the time of oocyte retrieval (6), and Gonadotropin-releasing hormone agonist (GnRHa) as an oocyte trigger in GnRH antagonist cycles (7), natural-cycle In Vitro Fertilization (IVF) or in vitro oocyte maturation (IVM) ${ }^{(8)}$.

Unfortunately, none of the strategies currently employed completely prevents OHSS after hCG administration ${ }^{(8)}$.

The risk of OHSS can be reduced by using a GnRHa trigger, instead of hCG trigger, in patients undergoing controlled ovarian stimulation (COS) with a GnRH-antagonist protocol. Since the technique was first suggested in $1988^{\left({ }^{(9)}\right.}$, a number of studies have investigated the efficacy and safety of a GnRHa trigger ${ }^{(9)}$.

An analysis by Humaidan et al. (10) demonstrated similar results in patients receiving a GnRHa trigger and those receiving an hCG trigger in terms of number of oocytes retrieved, fertilization 
rate and embryo quality score. However, patients receiving a GnRHa trigger had poor clinical outcomes, with a reduced likelihood of pregnancy and an extremely high early pregnancy loss rate, which was attributed to luteal phase insufficiency, despite standard luteal phase support (LPS). After GnRHa trigger the circulating levels of progesterone and estradiol are significantly lower throughout the luteal phase as compared to those obtained after hCG triggering due to the shorter half-life of luteinizing hormone) $\mathrm{LH}$ ( ( $\sim 60$ minutes) compared to that of hCG ( $>24$ hours).

Multiple strategies have been proposed to optimize luteal-phase function and improve pregnancy rates after GnRHa trigger ${ }^{(\mathbf{1 0})}$. Dual trigger of oocyte maturation using a fixed low dose of hCG $(1,000 \mathrm{IU})$ as an adjuvant to $\mathrm{GnRHa}$ appears to significantly improve implantation, clinical pregnancy and live birth rates in high responders with peak E2 $<4,000 \mathrm{pg} / \mathrm{mL}$ without increasing the risk of clinically significant OHSS ${ }^{(\mathbf{1 1})}$.

However, it still needs to be determined whether "dual-trigger", a single bolus of hCG or repeated low-doses of hCG is the best option. Regardless of the chosen protocol, it is crucial to individualize the luteal phase treatment with hCG according to the ovarian response to stimulation in an effort to reduce the risk of OHSS ${ }^{(11)}$.

Studies attempted to test the concept of the dual trigger, which involves combining GnRHa with a low dose of hCG to trigger oocyte maturation ${ }^{(12}$, 13). It was proposed that dual trigger approach provided better oocyte maturity and pregnancy rates $(12,13,14)$.

Our study attempts to assess if the dual trigger is more efficacious than the GnRH trigger alone in improves the pregnancy outcome without increase the incidence of OHSS and the efficacy of the dual trigger in obtaining a higher number of mature oocytes, higher numbers of embryos, higher fertilization rates and higher embryos quality.

\section{PATIENTS AND METHODS}

Study Design: This study is a single center RCT was performed at International Islamic Center for Population Studies and Research, Assisted Reproduction Unit, Al-Azhar University during the period from May 2016 to August 2018. A total of 150 infertile high responder women at risk of ovarian hyperstimulation syndrome (OHSS) who undergo intra-cytoplasmic sperm injection (ICSI) were recruited. The study was approved by University Medical Ethical Committee. An informed consent obtained from all patients included in this study.

\section{Participants}

The study included all high-risk patients who had one or more risk factors for developing OHSS such as: polycystic ovarian morphology (PCOM), polycystic ovarian syndrome (PCOS), or previous OHSS who underwent ICSI cycles using a GnRH antagonist protocol with peak E2 $>3,000 \mathrm{pg} / \mathrm{mL}$ and were triggered with either GnRHa alone or both GnRHa and 1,500 IU hCG for oocyte maturation. We excluded patients with one of the following conditions: older than 40 years, uterine abnormalities, poor responders to $\mathrm{COH}$ and $\mathrm{FSH}>$ $10 \mathrm{IU} / \mathrm{L}$.

\section{Treatment protocol}

Controlled ovarian stimulation was done according to the GnRH Antagonist protocol and initiated with highly purified urinary FSH (Fostimon, 75 I.U/150 I.U.; IBSA, Lugano) alone or with highly purified human Menopausal Gonadotropin (hMG) (Merional, 75 I.U/150 I.U.; IBSA, Lugano) in appropriate doses according to the age of the patient, FSH level, previous response and ovarian volume. Then serum E2 and ultrasound was done on day 6 of ovarian stimulation and step up or step down of gonadotrophin dose was adjusted according to E2 level and follicular activity. All patients underwent serial T.V.S assessment of follicular growth and measurement of serum E2 level every 1-3 days, based on the response and the need to evaluate the impact of any further adjustments in the dose of gonadotropin treatment.

$\mathrm{GnRH}$ antagonist cetrorelix (cetrotide) was started at $0.25 \mathrm{mg}$ subcutaneously daily once a follicle reached $\geq 14 \mathrm{~mm}$ in diameter or serum E2 reached $>350 \mathrm{pg} / \mathrm{mL}$ until the day of oocyte maturation trigger. Patients were triggered once two or more follicles reached $\geq 17 \mathrm{~mm}$ in diameter. Once the decision to trigger was taken, patients were allocated by the Computer-based randomization method into two groups:

- Group I (GnRHa trigger Group): included 75 women who received GnRHa trigger $(0.2 \mathrm{mg}$ triprtorelin acetate; decapeptyle) only subcutaneously for oocyte maturation.

- Group II (Dual trigger Group): includes 75 patients were trigger with $\mathrm{GnRHa}(0.2 \mathrm{mg}$ triprtorelin acetate; decapeptyle) plus 1,500 IU hCG subcutaneously for oocyte maturation.

Oocytes were retrieved by transvaginal ultrasound-guided needle aspiration. After aspiration, oocytes were screened then ICSI was done. Oocyte maturity and embryo grading were done as per the laboratory protocol, transvaginal ET 
was performed day 3 or day 5 (blastocyst) after oocyte retrival in both groups and patient was followed two weeks until pregnancy test. The remaining good quality embryos were freezed, transfer was canceled if the signs of OHSS (ultrasound evidence of ascites) were developed. All patients received luteal support with $400 \mathrm{mg}$ of progesterone pessaries (Cyclogest) $400 \mathrm{mg}$ twice daily from the day after oocyte retrieval until a negative pregnancy test or 10 weeks of gestation.

\section{Outcome measures}

The outcome measures were incidence and severity of OHSS, the oocyte maturation, fertilization rate, number of yield embryos, the quality of embryos, positive pregnancy test rate, clinical pregnancy, ongoing pregnancy, miscarriage, and implantation rate.

- The Fertilization rate was defined as how many oocytes become fertilized by sperm cells for each patient.

- Oocyte maturation rate was defined as the number of Metaphase II oocytes divided by the number of oocytes retrieved.

- The quality of embryos was calculated as the number of Grade I embryos divided by the number of yield embryos.

- Positive pregnancy test was defined by the rising serum beta subunit of human chorionic gonadotropin titers above $5 \mathrm{IU} / \mathrm{L}$ on the 14th day after retrieval.

- Clinical pregnancy was defined with the presence of fetal heart beat on transvaginal sonogram at 7 weeks of gestation.

- Ongoing pregnancies had surviving fetuses at 20 weeks gestation.
- Miscarriage was defined as the loss of the pregnancy until 20 weeks of gestation.

- The implantation rate was calculated as the number of beating fetal hearts divided by the number of embryos transferred per patient.

The diagnosis of OHSS was based on the criteria described by Mathur et al. ${ }^{(35)}$. Also OHSS was defined with an onset $\geq 10$ days after oocyte retrieval as "late" and OHSS with earlier onset as "early" OHSS.

\section{Data Analysis}

Data were checked entered and analyzed using SPSS version 22. Data were represented as mean \pm SD for quantitative variables, frequency and percentage for categorical variables. Chi- squared test, Fisher's exact test and t test were used when appropriate. $\mathrm{P}<0.05$ was considered statistically significant.

\section{RESULTS}

The two studied groups were matched for age, BMI, basal hormone levels, and cause of infertility. The mean age of the patients in GnRHa group versus dual group was $29.7 \pm 3.5$ years and $30.5 \pm 3.3$ years, respectively. The groups did not significantly differ with regard to the baseline characteristics such as age, BMI, duration of infertility and etiology of infertility in patients of both the groups (Table 1).

The basal FSH, LH, E2, Prolactin, the duration of stimulation and the average total dose of gonadotropins given to the patients did not differ between the groups compared (Table 1). There was no difference between the groups in the serum E2 levels on the day of triggering final oocyte maturation. 
Table (1): Demographic data, Comparison between Hormonal profile, average stimulation days and total number of ampoules used for stimulation and type of infertility and infertility cause

\begin{tabular}{|c|c|c|c|c|c|c|c|}
\hline & & \multicolumn{2}{|c|}{$\begin{array}{c}\text { Group I } \\
\text { GnRH agonist } \\
\text { trigger } \\
n=75\end{array}$} & \multicolumn{2}{|c|}{$\begin{array}{c}\text { Group II } \\
\text { Dual trigger } \\
n=75\end{array}$} & $\mathbf{t}$ & $\mathbf{p}$ \\
\hline & & \multicolumn{2}{|c|}{$\begin{array}{c}\mathrm{X} \pm \mathrm{SD} \\
\text { Range }\end{array}$} & \multicolumn{2}{|c|}{$\begin{array}{c}\mathrm{X} \pm \mathrm{SD} \\
\text { Range }\end{array}$} & & \\
\hline \multicolumn{2}{|l|}{ Age (years) } & \multicolumn{2}{|c|}{$\begin{array}{c}29.7 \pm 3.5 \\
(20-35) \\
\end{array}$} & \multicolumn{2}{|c|}{$\begin{array}{c}30.5 \pm 3.3 \\
(20-35)\end{array}$} & 0.69 & $0.49(\mathrm{Ns})$ \\
\hline \multicolumn{2}{|l|}{ BMI (kg/m2) } & \multicolumn{2}{|c|}{$\begin{array}{l}26.1 \pm 4.2 \\
(22-36)\end{array}$} & \multicolumn{2}{|c|}{$\begin{array}{l}27.0 \pm 3.6 \\
(22-35)\end{array}$} & 1.3 & $\begin{array}{l}0.17 \\
(\mathrm{Ns})\end{array}$ \\
\hline \multicolumn{2}{|c|}{ Duration of infertility (years) } & \multicolumn{2}{|c|}{$\begin{array}{c}5.1 \pm 3.4 \\
(1-15) \\
\end{array}$} & \multicolumn{2}{|c|}{$\begin{array}{c}5.4 \pm 2.6 \\
(1-16)\end{array}$} & 0.5 & $\begin{array}{l}0.59 \\
(\mathrm{Ns})\end{array}$ \\
\hline \multicolumn{2}{|l|}{ Serum FSH (mIU/ml) } & \multicolumn{2}{|c|}{$5.3 \pm 1.6$} & \multicolumn{2}{|c|}{$5.2 \pm 1.4$} & 0.2 & $0.8(\mathrm{Ns})$ \\
\hline \multicolumn{2}{|l|}{ Serum LH (mIU/ml) } & \multicolumn{2}{|c|}{$5.5 \pm 1.1$} & \multicolumn{2}{|c|}{$5.6 \pm 1.4$} & 0.2 & $0.8(\mathrm{Ns})$ \\
\hline \multicolumn{2}{|c|}{ Basal Estradiol E2(pg/ml) } & \multicolumn{2}{|c|}{$28.1 \pm 1.2$} & \multicolumn{2}{|c|}{$25.9 \pm 2.2$} & 0.17 & $\begin{array}{l}0.24 \\
(\mathrm{Ns}) \\
\end{array}$ \\
\hline \multicolumn{2}{|c|}{ Serum Prolactin (mIU/ml) } & \multicolumn{2}{|c|}{$18.6 \pm 3.5$} & \multicolumn{2}{|c|}{$18.2 \pm 3.0$} & 0.25 & $\begin{array}{l}0.79 \\
(\mathrm{Ns})\end{array}$ \\
\hline \multicolumn{2}{|c|}{ Serum Estradiol (E2) on day of trigger (pg/ml) } & \multicolumn{2}{|c|}{$4219.8 \pm 178$} & \multicolumn{2}{|c|}{$4273.3 \pm 101$} & 0.23 & $\begin{array}{l}0.81 \\
(\mathrm{Ns})\end{array}$ \\
\hline \multicolumn{2}{|c|}{$\begin{array}{l}\text { Mean number of ampoules used for stimulation per } \\
\text { day }\end{array}$} & \multicolumn{2}{|c|}{$3.9 \pm 0.6$} & \multicolumn{2}{|c|}{$3.6 \pm 0.2$} & 1.4 & $\begin{array}{l}0.16 \\
(\mathrm{Ns})\end{array}$ \\
\hline \multicolumn{2}{|c|}{ Mean days of stimulation } & \multicolumn{2}{|c|}{$\begin{array}{c}11.2 \pm 1.7 \\
(8-19)\end{array}$} & \multicolumn{2}{|c|}{$\begin{array}{c}11.18 \pm 1.7 \\
(8-17)\end{array}$} & 0.04 & $\begin{array}{l}0.96 \\
(\mathrm{Ns})\end{array}$ \\
\hline \multirow{2}{*}{\multicolumn{2}{|c|}{ Mean total number of ampoules }} & & & $\begin{array}{r}39.9 \\
(15 \\
\end{array}$ & $\begin{array}{ll}6.7 \\
1)\end{array}$ & 0.5 & $\begin{array}{l}0.57 \\
(\mathrm{Ns})\end{array}$ \\
\hline & & No. & $\%$ & No. & $\%$ & $\mathbf{X}^{2}$ & $\mathbf{P}$ \\
\hline Type of infertility & $\begin{array}{l}\text { - Primary } \\
\text { - Secondary }\end{array}$ & $\begin{array}{l}31 \\
44 \\
\end{array}$ & $\begin{array}{l}41.3 \\
58.7\end{array}$ & $\begin{array}{l}35 \\
40\end{array}$ & $\begin{array}{l}46.7 \\
53.3\end{array}$ & 0.43 & $0.5(\mathrm{Ns})$ \\
\hline Cause of infertility & - Ovarian factor only & 39 & 49.3 & 43 & 57.3 & 0.43 & $0.5(\mathrm{Ns})$ \\
\hline & - Multiple factors & 20 & 26.7 & 21 & 28.0 & 0.03 & $0.8(\mathrm{Ns})$ \\
\hline & - Tubal factor & 9 & 12.0 & 7 & 9.3 & 0.28 & $0.6(\mathrm{Ns})$ \\
\hline & - Unexplained infertility & 7 & 9.3 & 4 & 5.3 & 0.88 & $0.3(\mathrm{Ns})$ \\
\hline No. of patients with $P$ & & 59 & 78.7 & 64 & 85.3 & 1.13 & $0.2(\mathrm{Ns})$ \\
\hline
\end{tabular}

Although the average number of total oocytes retrieved $(12.8 \pm 3.6$ vs. $13.6 \pm 3.8 ; \mathrm{P}=$ $0.18)$ and the fertilization rate $(62.1 \pm 11$ vs. $65.3 \pm$ $10.1 ; \mathrm{P}=0.06)$ were more in the dual group as compared to GnRHa group, the difference were insignificant.
The dual-trigger group had a significantly higher in the number of mature oocytes recovered $(10.2 \pm 3.3$ vs. $12.3 \pm 4.2 ; \mathrm{P}<0.001)$, the number of yield embryos $(6.2 \pm 2.4$ vs. $7.8 \pm 2.8 ; \mathrm{P}<0.001)$, also and high statistically significant differences regarding to the quality of embryos $(54.19 \%$ vs. $63.76 \%$; $\mathrm{P}$ < 0.001) compared with the GnRHa trigger group. 
Table (2): Comparison between the studied groups as regards the number of follicles, number of oocytes retrieved, number of Metaphase II oocytes, number of yield embryos, fertilization rate and quality of embryos.

\begin{tabular}{lcccc}
\hline Mean number of follicles & Group I & Group II & $\mathbf{t}$ & P \\
Range & $\begin{array}{c}15.1 \pm 3.9 \\
(8-31)\end{array}$ & $\begin{array}{c}16.2 \pm 4.2 \\
(7-37)\end{array}$ & 1.6 & $0.09(\mathrm{Ns})$ \\
\hline Mean number of oocytes retrieved & $\begin{array}{c}12.8 \pm 3.6 \\
(5-22)\end{array}$ & $\begin{array}{c}13.6 \pm 3.8 \\
(5-25)\end{array}$ & 1.3 & $0.18(\mathrm{Ns})$ \\
Range & $\begin{array}{c}10.2 \pm 3.3 \\
(3-18)\end{array}$ & $\begin{array}{c}12.3 \pm 4.2 \\
(4-23)\end{array}$ & 3.4 & $<0.001(\mathrm{Hs})$ \\
\hline Mean number of Metaphase II oocytes & $\begin{array}{c}6.2 \pm 2.4 \\
(2-16)\end{array}$ & $\begin{array}{c}7.8 \pm 2.8 \\
(2-21)\end{array}$ & 3.7 & $<0.001(\mathrm{Hs})$ \\
\hline Mange & $\begin{array}{c}62.1 \pm 11.0 \\
(20-88.9)\end{array}$ & $\begin{array}{c}65.3 \pm 10.1 \\
(22-91.3)\end{array}$ & 1.85 & $0.06(\mathrm{Ns})$ \\
\hline Range & $\begin{array}{c}252 / 465 \\
(54.19)\end{array}$ & $373 / 585(63.76)$ & 9.84 & $<0.0017(\mathrm{Hs})$ \\
\hline Quality of embryos & & & & \\
\hline
\end{tabular}

Our study showed that the dual trigger (Group II) improved positive pregnancy test rate $(53.3 \%$ vs $36 \%, \mathrm{p}<0.05)$, clinical pregnancy $(48 \%$ vs $28 \%, \mathrm{p}<0.05)$ and ongoing pregnancy rates $(44 \%$ vs $20 \%, \mathrm{p}<0.05)$ compared with GnRHa group (Group I) with statistically significant difference between the two groups. Also, showed that dual trigger decreased the rate of chemical pregnancy $(10 \%$ vs $22.2 \%, p>0.05)$, miscarriage $(7.5 \%$ vs $22.2 \%, \mathrm{p}>0.05$ ) compared to GnRHa alone but with no statistical significant difference. The implantation rate in dual trigger group (47.7\% vs $33.8 \%, \mathrm{p}>0.05$ ) and the rate of multiple pregnancy $(8.0 \%$ vs $2.7 \%$, p $>0.05$ ) were higher than GnRHa trigger
Group but there were statistically insignificant between the groups.

There were 6 case of OHSS developed with dual trigger group (Group II) (3 were mild early OHSS, 2 were moderate early OHSS and one case was severe late OHSS). In contrast, there was only one case of severe late OHSS seen in Group I so the incidence of OHSS was higher after dual trigger than GnRHa trigger but but with no statistically significance $(8.0 \%$ vs $1.33 \%, \mathrm{p}>0.05$ ).

The cases of early OHSS in dual trigger group were 5 but there was no case of early OHSS developed in $\mathrm{GnRH}$ a trigger group and there was one case of severe late OHSS developed in each group (Table 3). 
Table (3): Comparison between the studied groups as regards the rate of positive pregnancy test, Chemical pregnancy, Clinical pregnancy, Miscarriage, Ongoing pregnancy, Multiple pregnancy and implantation rate. Comparison between the studied groups as regards OHSS occurrence, severity and types

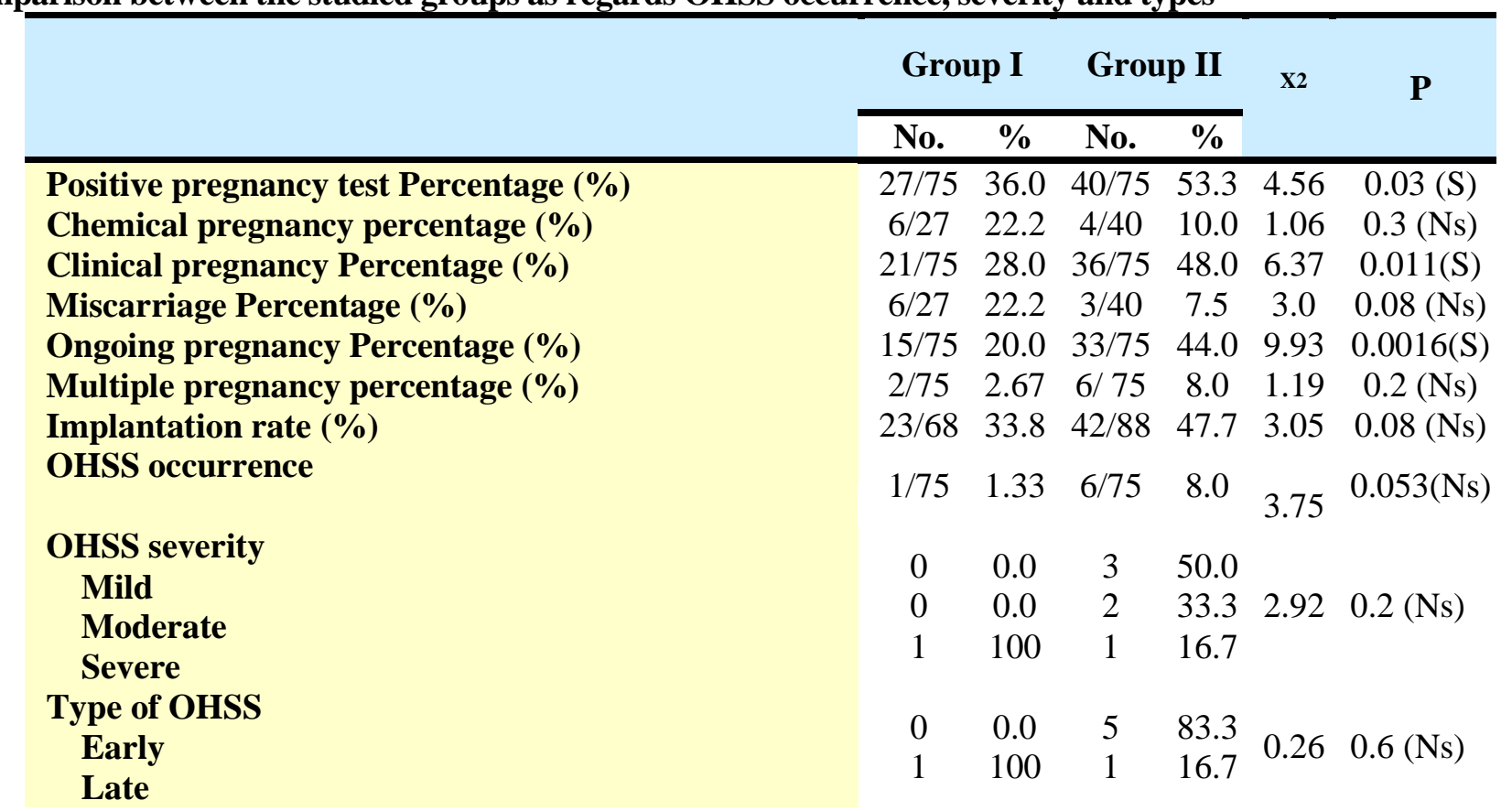

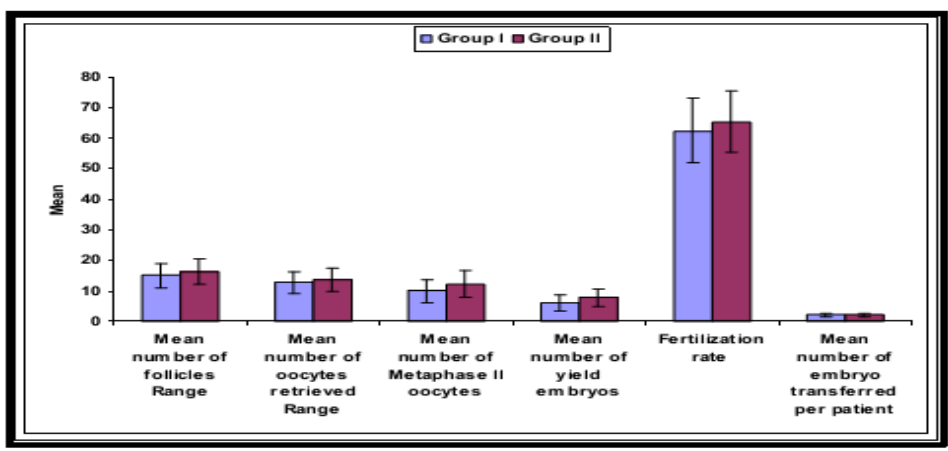

Fig (1): The studied groups as regards the number of follicles, number of oocytes retrieved, number of Metaphase II oocytes, number of yield embryos, fertilization rate and number of embryo transferred per patient (ET).
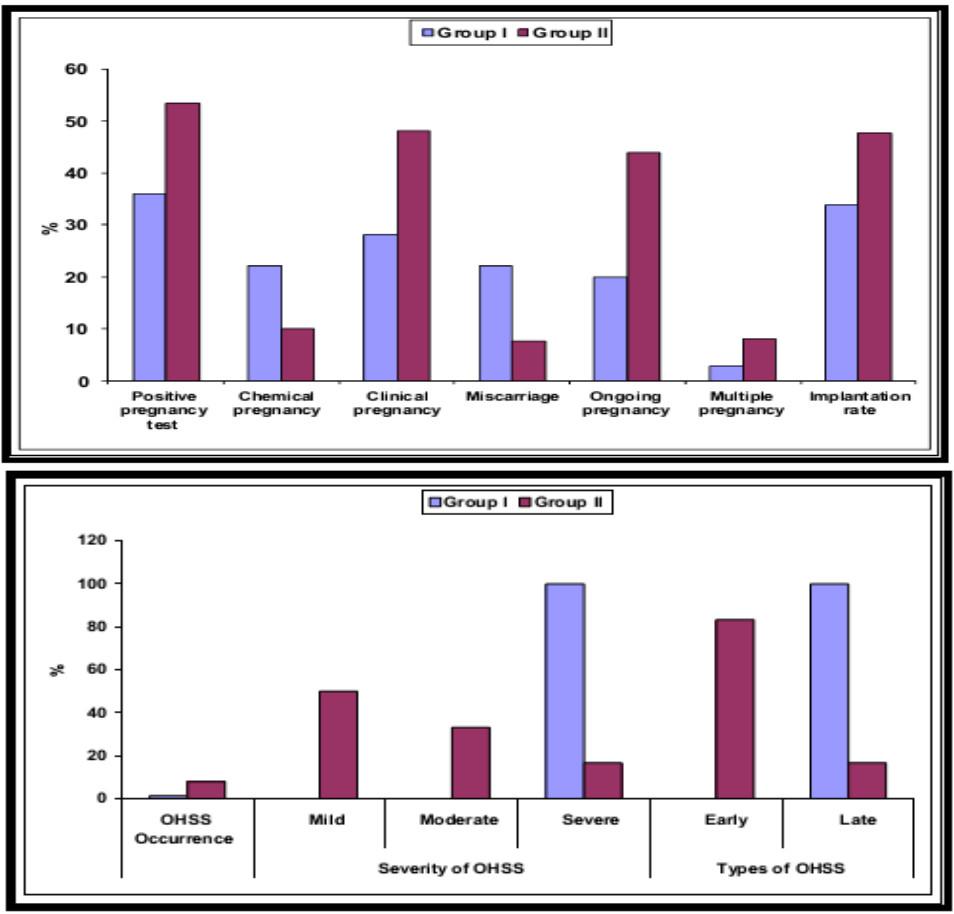

Fig (3): The studied groups as regards OHSS occurrence, severity and the types of OHSS 


\section{DISCUSSION}

Ovarian hyperstimulation syndrome (OHSS) remains the most challenging complication in assisted reproductive technology, which potentially puts the patient into life-threatening danger ${ }^{(15)}$.

Although there have been several strategies proposed to decrease the incidence and the severity of OHSS, GnRH agonist (GnRHa) to induce oocyte maturation has been shown to be the most effective method to reduce the risk of OHSS ${ }^{(16,17)}$. Studies have shown lower conception and higher miscarriage rates in patients triggered with $\mathrm{GnRHa}$ compared with $\mathrm{hCG}^{\mathbf{1 8}}$, 19).

Our study showed that the incidence of OHSS was higher after dual trigger than GnRHa trigger but with no statistically significant difference (8.0 vs $1.33 \%$, p $>0.05$ ). There were 6 case of OHSS developed with dual trigger group (Group II), 5 of them were early and one was severe late OHSS. In contrast, there was only one severe late OHSS seen in Group I. so the dual trigger increased the incidence of early OHSS and not increase the late type.

The results of this study found no significant differences in the number of follicles and the number of oocytes retrieval between Group I receiving GnRH a alone and Group II receiving the dual trigger but there were high statistically significant difference $(\mathrm{p}<0.001)$ as regards the oocyte maturity (Metaphase II oocytes) and the number of yield embryos which were lower in Group I than in Group II.

The fertilization rate was slightly higher in Group II than Group I but the differences were statistically insignificant between the two groups ( $p>0.05$ ). The study showed high significant difference regarding to the quality of embryos yield which was higher in dual trigger group $(\mathrm{p}<0.001)$.

Our study showed that the dual trigger of final oocyte maturation with GnRHa and low-dose hCG (Group II) resulted in improved positive pregnancy test, clinical pregnancy, ongoing pregnancy rates compared with GnRHa trigger alone (Group I) with statistically significant difference between the two groups $(\mathrm{p}<0.05)$. Also, showed that dual trigger decreased the rates of chemical pregnancy and spontaneous miscarriage compared to GnRHa trigger alone but with statistically insignificant difference between the two groups, the rate of multiple pregnancy and implantation were higher in dual trigger group than $\mathrm{GnRHa}$ trigger group but insignificantly.

O'Neill et al. ${ }^{(20)}$ performed a retrospective cohort study at an academic center to compare rates of OHSS after using GnRHa alone and GnRHa in combination with low-dose hCG (dual trigger) for final oocyte maturation in women undergoing $\mathrm{CoH}$, the study demonstrated that dual trigger for final oocyte maturation using GnRHa and low-dose hCG (1000 IU) is associated with a significantly increased the incidence of early OHSS than GnRHa trigger (8.6 vs $0 \%, \mathrm{p}<0.01)$ and our study finding also showed increased the incidence of early OHSS but insignificantly, in o'Neill study the majority of these patients (four of six) were diagnosed with severe OHSS this was not agreed with our findings as the majority of our OHSS cases were mild and moderate degree. So they concluded that dual trigger also a significantly increased risk of severe OHSS compared to GnRHa alone. They found that Dual trigger was associated with a higher number of oocytes obtained but with no statistically significant difference and oocyte maturity was higher after dual trigger than trigger with GnRHa alone (82 vs $70 \%$, $\mathrm{p}<0.01)$ and they found that there was no differences in the fertilization rates between groups so those results agreed with our study. There was a trend towards improved pregnancy rate, there were no statistically significant differences in the rates of clinical pregnancy (44 vs $63 \%, p=0.12$ ) or spontaneous miscarriage ( 7 vs $6 \%, \mathrm{p}=0.90$ ) in the GnRHa trigger group compared to the dual trigger group but the rate of clinical pregnancy in our study was higher in dual trigger group with statistically significant difference.

Huang et al. ${ }^{(21)}$ conducted a retrospective study at a tertiary fertility center in Taiwan to assess the pregnancy outcome and OHSS incidence in high responders receiving GnRHa trigger plus individualized support of low-dose hCG. The study showed that none of the patients in dual trigger Group and GnRHa trigger Group developed moderate/ severe OHSS and this finding was different from our finding. There is no significant difference in the oocyte maturity between the GnRHa trigger group and the dual trigger group which was statistically significant in our study. Also, they found a higher fertilization rate in dual trigger Group (mean 82.2\%), significantly when compared with GnRHa trigger Group (mean 68.5\%) but in our study fertilization rate was slightly higher in dual trigger Group than GnRHa trigger Group but with no significant difference. There was a tendency toward a better outcome in dual trigger Group than GnRHa trigger Group in terms of higher implantation rate $(22.5 \pm 5.2 \%$ vs. $5.8 \pm 2.8 \%)$, clinical pregnancy rate (48.5\% vs. $17.4 \%)$ and ongoing pregnancy rate ( $46.9 \%$ vs. $17.4 \%$ ) as well as lower miscarriage rate ( $16.7 \%$ vs. $50.0 \%$ ); but probably due to the limited case number, it did not reach statistical significance. 
A retrospective cohort study, a systematic review with data collected from electronic medical records of a Human Reproduction Clinic in Spain were carried out by De Oliveira et al. ${ }^{(22)}$ for high responder patients who had been treated with Dual Trigger. Dual Triggering has been compared to $\mathrm{GnRH}$ agonist triggering, the group of high responders consisted of a total of 24 patients, 12 in group A (Dual Trigger) and 12 in B (GnRH alone). There was no statistically significant difference among collected oocyte numbers, fertilized embryos, transferred embryos, or vitrified embryos, number of mature oocytes and good quality embryos but last two items were statistically significant high in dual trigger group in our study. Group A (Dual Trigger) showed higher implantation and newborn/transferred embryo rates with statistical significance which was higher in our study but insignificant. Their retrospective study and systematic review demonstrated that there is yet no indication of the use of Dual Triggering mode in high responder patients, since the results of those studies were contradictory, and it was impossible to assess the risk of OHSS due to the low prevalence of this disorder and that conclusion wasn't agree with our study.

Another group of investigators (11) performed a Retrospective cohort study to compare live birth rates after dual trigger of oocyte maturation with GnRHa and low-dose hCG versus GnRHa alone in high responders at risk of OHSS, the study showed that The dual-trigger group had a significantly higher live birth rate $(52.9 \%$ vs. $30.9 \%)$, implantation rate ( $41.9 \%$ vs. $22.1 \%)$, and clinical pregnancy rate (58.8\% vs. $36.8 \%$ ) compared with the GnRHa trigger group. one case of mild OHSS occurred in the dual-trigger group, and there were no cases of OHSS in the GnRHa trigger group, but in our study there were 6 cases of OHSS with dual trigger group (Group II) (3 were early mild OHSS, 2 were early moderate and only one case was severe late OHSS) and there was one case of late severe OHSS was seen in Group I.

Shapiro and colleagues ${ }^{(\mathbf{1 2})}$ did retrospective study of fresh autologous blastocyst transfers in high responders compared ongoing pregnancy rates in cycles that followed trigger with $\mathrm{GnRH}$ agonist alone with standard luteal support, GnRHa alone with enhanced luteal support, or GnRHa with concomitant low-dose hCG (dual trigger). The incidence of clinically significant ovarian hyperstimulation syndrome was $0.0 \%$ in the groups receiving only GnRHa and $0.5 \%$ (1 of 182) in patients receiving GnRHa with concomitant low-dose $\mathrm{hCG}$ and this percentage is less than our study. The lowdose hCG with a GnRH agonist trigger was associated with much greater ongoing pregnancy and implantation rates and reduced pregnancy loss rates when compared with the agonist trigger alone and the difference were statistically significant. In our study the dual trigger was associated with greater implantation rates and reduced pregnancy loss rates compared to GnRHa trigger alone but the difference were statistically insignificant.

Shapiro et al. ${ }^{(13)}$ performed a retrospective study to evaluate the effect of administration of a low dose of hCG in combination with leuprolide acetate to induce final oocyte maturation in patients who have significant risk factors for OHSS. The results of 45 retrievals after dual trigger showed that each cycle had $>11$ retrieved oocytes, none was canceled, and all achieved transfer of one to two blastocysts. of these, 29 had positive pregnancy tests, but 5 pregnancies were lost by 10 weeks of gestation. The early pregnancy loss rate was therefore $5 / 29$, or $17.2 \%$ of pregnancies, and the ongoing pregnancy rate was $53.3 \%$ per transfer. No patient was diagnosed with even mild OHSS, despite many of them having high-risk factors. But in our study, we had 6 patients got OHSS. But in our study, we had 6 patients got OHSS.

With the introduction of the GnRH antagonist protocol for the prevention of a premature LH surge, it became possible to trigger ovulation with GnRHa. The GnRH antagonist occupies the GnRH receptor without causing down-regulation, and by injecting a single bolus of GnRHa, the antagonist is displaced from the receptor. This activates the receptor, inducing a flare-up of gonadotrophins ( $\mathrm{LH}$ and FSH), which effectively stimulate the final oocyte maturation and ovulation. However, important differences exist regarding the profile and duration of the LH surge after triggering with GnRHa compared to that of the natural cycle. In the natural cycle, the LH surge is characterized by three phases with a total duration of $\sim 48$ hours. After GnRHa triggering, the surge consist of two phases, only, with a duration of 24-36 hours leading to a significantly reduced amount of LH released ${ }^{(10)}$. The short half-life of pituitary LH combined with the desensitization induced by the agonist results in a rapid and irreversible luteolysis, ideally eliminating the risk of OHSS ${ }^{(12,11)}$. So the use of GnRH agonist triggering has been reported as an effective method to prevent clinically significant OHSS in high responders ${ }^{(18,23}$, 24). In a recent study, the expression of vascular endothelial growth factor (VEGF) and inhibin $\beta$ was found to be lower in the granulosa cells collected from patients with $\mathrm{GnRH}$ agonist triggering than those from patients with hCG triggering, which may be another factor for the lower OHSS rate ${ }^{(25)}$.

In addition to that, some studies have shown that administering $\mathrm{GnRH}$ agonist after the use of $\mathrm{GnRH}$ antagonist in an IVF cycle brings about true benefits for implantation, since the antagonist blocks 
endometrial $\mathrm{GnRH}$ receptors, worsening endometrial quality. once the GnRH agonist that has a much higher affinity to receptor than the $\mathrm{GnRH}$ antagonist is administered, a displacement of the antagonist from the receptor occurs in the endometrium, and it unlocks these receptors, improving endometrial receptivity ${ }^{(26)}$

Apart from an LH surge, GnRHa triggering also induces an initial secretion of FSH resembling that of the natural cycle. This more natural surge of gonadotrophins after triggering with GnRHa may explain why some authors reported retrieval of an increased amount of mature oocytes compared to hCG triggering ${ }^{(10,27)}$.

The induced surge of gondotrophins results in an initial rise in the levels of progesterone and estradiol followed by a decrease during the next 24 hours prior to oocyte pick-up (OPU). Subsequently a second rise in the level of progesterone takes place as ovarian steroidogenesis shifts from follicular to luteal phase. In contrast, the estradiol level continues to fall. After GnRHa trigger the circulating levels of progesterone and estradiol are significantly lower throughout the luteal phase as compared to those obtained after hCG triggering due to the shorter half-life of LH $(\sim 60$ minutes) compared to that of hCG (>24 hours) ${ }^{(\mathbf{1 0})}$. The important clinical advantage of GnRHa triggering, however, is the reported significant reduction in or even elimination of OHSS ${ }^{(\mathbf{1 0}, 27)}$ caused by the shorter halflife of the endogenous LH surge compared with the continuous $\mathrm{LH} / \mathrm{hCG}$ receptor stimulation after hCG triggering ${ }^{(10,28)}$.

The luteal phase after $\operatorname{CoS}$ is defect due to supraphysiological steroid hormone concentrations inhibiting the LH secretion via negative feedback at the level of the hypothalamic-pituitary-gonadal-axis, the LH activity will be further compromised after GnRHa triggering due to the shorter duration of the endogenous induced LH surge and a potential weaker activation of the $\mathrm{LH} / \mathrm{hCG}$ receptor. The result of this is a significant reduction in LH activity throughout the early/mid luteal phase leading to premature luteolysis and implantation failure. In contrast, after hCG triggering, the luteal actions of LH will be covered by the bolus of hCG injected and then gradually by the hCG produced by the implanting embryo ${ }^{(\mathbf{1 0})}$.

So the rapid luteolysis caused by the use of the agonist consequently leads to an altered luteal phase, and its final result is the reduction of implantation rates and the increasing of miscarriage rates, when compared to the use of hCG as a "trigger" ${ }^{29,11)}$. To solve this problem, some studies have shown that it was possible to improve implantation rates by administering high doses of progesterone alone or combined with estrogen on the luteal phase after using the GnRH agonist ${ }^{(12,11)}$. Another possibility would be to transfer the vitrification of embryos into another cycle with more appropriate hormone levels. Furthermore, in an effort to reduce miscarriage rates, few studies have evaluated the effects of the use of GnRH agonists associated with hCG 12-35 hours after triggering with the agonist, and they have shown some improvements in the luteal phase ${ }^{(30)}$. However, the subsequent hCG administration does not act as oocyte maturation ${ }^{(\mathbf{1 3})}$.

For approximately eight years, some studies have shown a possibility of the combination of the use of GnRH agonist concurrently with hCG to induce final oocyte maturation (11), a treatment known as "Dual Trigger", which has been used in patients with high response, normal response, and poor response, or oocyte immaturity ${ }^{(\mathbf{1 1})}$.

Theoretically, this method combines the advantages of both induction regimens:

1. It decreases the risk of OHSS by decreasing the dose of hCG;

2. It tends to be a more physiological cycle since there is an FSH peak addition induced by $\mathrm{GnRH}$ agonist generating a larger number of mature oocytes ${ }^{(31,25,32)}$, whereas hCG alone induces LH peak ${ }^{(33)}$.

3. It improves endometrial receptivity for releasing endometrial GnRH receptors.

4. It extends the ovulation time after use of the inductor caused by hCG, also improving the maturation ${ }^{(34)}$.

5. There is better luteal phase recruitment when there is a proven combined use of hCG with GnRH agonist ${ }^{(\mathbf{1 2})}$.

In our study, the use of hCG in the Dual trigger incurs some risk of clinically significant OHSS as 6 cases were observed in the dual-trigger group. However, the Dual Trigger used for induction of final oocyte maturation significantly improved the beta hCG positive rate, clinical pregnancy and ongoing pregnancy rate. Also, the dual trigger significantly improved oocyte maturity and the quality of embryos.

\section{CONCLUSION}

The study showed that Dual trigger for final oocyte maturation using GnRHa and low-dose hCG is associated with a significantly increased incidence of OHSS compared to GnRHa alone. However, dual trigger may be associated with a significant increase in the beta hCG positive rate, clinical pregnancy and ongoing pregnancy rate in high responders and reduce the rate of miscarriage compared with GnRHa alone. 
It is recommended that larger prospective randomized controlled studies are needed to evaluate the optimal timing and dosage of hCG to be given with GnRHa trigger to maintain adequate pregnancy rates without increasing the risk of clinically significant OHSS.

\section{REFERENCES}

1- Mathur R, Kailasam C and Jenkins J (2007): Review of the evidence base strategies to prevent ovarian hyperstimulation syndrome. Hum Fertil., 10: 75-85.

2- Gómez R, Soares SR, Busso C et al. (2010): Physiology and pathology of ovarian hyperstimulation syndrome. Semin Reprod Med., 28:448-457.

3- Chen CD, Chao KH, Yang JH et al. (2010): Comparison of coasting and intravenous albumin in prevention of ovarian hyperstimulation syndrome. Fertil Steril., 80:86-90.

4- Gracia-Velasco JA (2009): How to avoid ovarian hyperstimulation syndrome: a new indication for dopamine agonists. Reprod Biomed Online, 18: 71-5.

5- Gracia-Velasco JA, Isaza V, Quea G et al. (2006): Coasting for the prevention of ovarian hyperstimulation syndrome: much ado about nothing? Fertil Steril., 85: 547554.

6- Aboulghar MA, Mansour RT, Amin YM et al. (2009): A prospective randomized study comparing coasting with $\mathrm{GnRH}$ antagonist administration in patients at risk for severe OHSS. Reproductive BioMedicine Online, 15: 271279.

7- Youssef M, Van der veen F, AL-inany $\mathrm{H}$ et al. (2009): Gonadotropin-releasing hormone agonist versus HCG for oocyte triggering in antagonist ART cycles. https://www.cochranelibrary.com/cdsr/doi/10.1002/146518 58.CD008046.pub4/.../full

8- Edwards RG (2007): IVF, IVM, natural cycle IVF, minimal stimulation. Reprod Biomed Online, 15: 106-119.

9- Itskovitz-Eldor J, Kol S, Mannaerts B et al. (1998): First established pregnancy after controlled ovarian hyperstimulation with recombinant follicle stimulating hormone and the gonadotrophin-releasing hormone antagonist ganirelix . Hum Reprod., 13:294-5.

10- Humaidan P, Kol S and Papanikolaou EG (2011): $\mathrm{GnRH}$ agonist for triggering of final oocyte maturation: time for a change of practice? Hum Reprod Update, 17(4): 510-524.

11- Griffin D, Benadiva C, Kummer N et al. (2012): Dual trigger of oocyte maturation with gonadotropin-releasing hormone agonist and low-dose human chorionic gonadotropin to optimize live birth rates in high responders. Fertil Steril., 97:1316-20.

12- Shapiro BS, Daneshmand ST, Restrepo H et al. (2011): Efficacy of induced luteinizing hormone surge after "trigger" with gonadotropin-releasing hormone agonist. Fertil Steril., 95:826-8.

13- Shapiro BS, Daneshmand ST, Garner FC et al. (2008): Gonadotropin-releasing hormone agonist combined with a reduced dose of human chorionic gonadotropin for final oocyte maturation in fresh autologous cycles of in vitro fertilization. Fertil Steril., 90:231-3.
14- Orvieto $\mathbf{R}$ (2015): Triggering final follicular maturation - HCG, GnRH-agonist or both, when and to whom? J Ovarian Res., 8:60-63.

15- Humaidan $P$, Ejdrup Bredkjaer $H$, Westergaard LG et al. (2010): 1,500 IU human chorionic gonadotropin administered at oocyte retrieval rescues the luteal phase when gonadotropin-releasing hormone agonist is usedfor ovulation induction: a prospective, randomized, controlled study. Fertil Steril., 93:847-54.

16- DiLuigi AJ, Engmann L, Schmidt DW et al. (2010): Gonadotropin-releasing hormone agonist to induce final oocyte maturation prevents the development of ovarian hyperstimulation syndrome in high-risk patients and leads to improved clinical outcomes compared with coasting. Fertil Steril., 94:1111-4.

17- Engmann L, DiLuigi A, Schmidt D et al. (2008): The use of gonadotropin-releasing hormone $(\mathrm{GnRH})$ agonist to induce oocyte maturation after cotreatment with GnRH antagonist in high-risk patients undergoing in vitro fertilization prevents the risk of ovarian hyperstimulation syndrome: A prospective randomized controlled study. Fertil Steril., 89:84-91.

18- Humaidan $\mathrm{P}$, Bredkjaer HE, Bungum $\mathrm{L}$ et al. (2005): GnRH agonist (buserelin) or hCG for ovulation induction in GnRH antagonist IVF/ICSI cycles: a prospective randomized study. Hum Reprod., 20: 1213-20.

19- Kolibianakis EM, Schultze-Mosgau A et al. (2005): A lower ongoing pregnancy rate can be expected when $\mathrm{GnRH}$ agonist is used for triggering final oocyte maturation instead of HCG in patients undergoing IVF with GnRH antagonists. Hum Reprod., 20:2887-92.

20- O'Neill KE, Senapati S, Maina I et al. (2016): GnRH agonist with low-dose hCG (dual trigger) is associated with higher risk of severe ovarian hyperstimulation syndrome compared to GnRH agonist alone. J Assist Reprod Genet., 33:1175-1184.

21- Huang C-Y, Shieh M-L and Li H-Y (2016): The benefit of individualized low-dose hCG support for high responders in GnRHa-triggered IVF/ICSI cycles. J Chinese Medical Association, 79 :387-393.

22- De Oliveira SA, Calsavara VF and Cortés GC (2016): Final Oocyte Maturation in Assisted Reproduction with Human Chorionic Gonadotropin and Gonadotropin-releasing Hormone agonist (Dual Trigger): JBRA Assist Reprod., 20(4): 246-250.

23- Itskovitz-Eldor J, Kol S, and Mannaerts B (2000): Use of a single bolus of GnRH agonist triptorelin to trigger ovulation after $\mathrm{GnRH}$ antagonist ganirelix treatment in women undergoing ovarian stimulation for assisted reproduction, with special reference to the prevention of ovarian hyperstimulation syndrome: preliminary report: short communication. Hum Reprod., 15: 1965-1968.

24- Engmann L, Siano L, Schmidt D et al. (2006): GnRH agonist to induce oocyte maturation during IVF in patients at high risk of OHSS. Reprod Biomed Online, 13: 639-644.

25- Haas J, Ophir L, Barzilay E et al. (2014): GnRH agonist vs. hCG for triggering of ovulation differential 
effects on gene expression in human granulosa cells. PLoS One, 9: 90-95.

26- Schachter M, Friedler S, Ron-El R et al. (2008): Can pregnancy rate be improved in gonadotropin-releasing hormone $(\mathrm{GnRH})$ antagonist cycles by administering $\mathrm{GnRH}$ agonist before oocyte retrieval? A prospective, randomized study. Fertil Steril., 90:1087-93.

27- Humaidan P, Papanikolaou EG, Kyrou D et al. (2012): The luteal phase after GnRH-agonist triggering of ovulation: present and future perspectives. Reprod Biomed Online, 24(2): 134-141.

28- Fauser BC, de Jong D, Olivennes F et al. (2002): Endocrine profiles after triggering of final oocyte maturation with $\mathrm{GnRH}$ agonist after cotreatment with the $\mathrm{GnRH}$ antagonist ganirelix during ovarian hyperstimulation for in vitro fertilization. J Clin Endocrinol Metab., 87(2): 709-715.

29- Lin MH, Wu FS, Lee RK et al. (2013): Dual trigger with combination of gonadotropin-releasing hormone agonist and human chorionic gonadotropin significantly improves the live-birth rate for normal responders in GnRHantagonist cycles. Fertil Steril., 100:1296-302.

30- Humaidan $P$ (2009): Luteal Phase rescue in high-risk OHSS patients by GnRHa triggering in combination with low-dose HCG: a pilot study. Reprod Biomed Online, 18:630-4.

31- Griffin D, Feinn R, Engmann L et al. (2014): Dual trigger with gonadotropin-releasing hormone agonist and standard dose human chorionic gonadotropin to improve oocyte maturity rates. Fertil Steril., 102:405-9.

32- Castillo JC, Moreno J, Dolz M et al. (2013): Successful Pregnancy Following Dual Triggering Concept (rhCG + GnRH Agonist) in a Patient Showing Repetitive Immature Oocytes and Empty Follicle Syndrome: Case Report. J Med Cases, 4:221-6.

33- Decleer W, Osmanagaoglu $\mathrm{K}$, Seynhave $\mathrm{B}$ et al. (2014): Comparison of hCG triggering versus hCG in combination with a GnRH agonist: a prospective randomized controlled trial. Facts Views Vis Obgyn., 6:203-9.

34- Zilberberg E, Haas J, Dar S et al. (2015): Coadministration of GnRH-agonist and hCG, for final oocyte maturation (double trigger), in patients with low proportion of mature oocytes. Gynecol Endocrinol., 31: 145-7.

35- Mathur R, Evbuomwan I and Jenkins J (2005): Prevention and management of ovarian hyperstimulation syndrome. Current Obstet Gynaecol., 15:132-8. 